FACTA UNIVERSITATIS (NIŠ)

Ser. Math. Inform. Vol. 35, No 3 (2020), 789-800

https://doi.org/10.22190/FUMI2003789B

\title{
SPACELIKE TRANSLATION SURFACES IN MINKOWSKI 4-SPACE $\mathbb{E}_{1}^{4}$
}

\author{
Sezgin Büyükkütük and Günay Öztürk
}

\begin{abstract}
(C) by University of Niš, Serbia | Creative Commons Licence: CC BY-NC-ND Abstract. In the present paper, we consider spacelike translation surfaces in 4-dimensional Minkowski space. We characterize such surfaces in terms of their Gaussian curvature and mean curvature functions. We classify flat and minimal spacelike translation surfaces in $\mathbb{E}_{1}^{4}$.
\end{abstract}

Keywords: spacelike translation surfaces; Minkowski space; Gaussian curvature.

\section{Introduction}

A surface's geometry consists of some properties like area, distance, angle and curvature. The most important of these is curvature, which reveals the structural differences between surfaces. Flat and minimal surfaces with zero Gaussian and zero mean curvature have major significance in geometry. Especially, a minimal surface is a surface that locally minimizes its area. In addition to the planes, catenoids and helicoids, the appearance of minimal surfaces can also be observed in nature: in the structures that animals build, in various plants and animal anatomies, etc. In history, some mathematicians such as Riemann, Schwarz, Scherk, Weierstrass and Enneper made major advances on minimal surfaces (see, [18]). During 1960s, the pioneering work of Osserman influenced the majority of modern theories of minimal surfaces in three dimensional spaces [17]. Minimal surfaces have also been the subject of today's work (see, [16]).

A special surface: Translation surface which is known as double curved in differential geometry are base for roofing structures. The construction and design of freeform glass roofing structures are generally created with the help of curved (formed) glass panes or planar triangular glass facets. Especially, double curved surface are made up of quadrilateral, that is four sided, facets. They lead to economic advantages compared to triangular glass facets. Because of these advantages, translation surfaces are used to construct free form glass roofing structures [11]. Also,

Received September 25, 2019; accepted January 21, 2020

2010 Mathematics Subject Classification. Primary 53A05; Secondary 53A10 
due to geometric property of these surfaces, they are used for Teichmüller theory in physics (see, [9]).

Translation surfaces can be parameterized locally as $\phi(u, v)=(u, v, f(u)+g(v))$. In [4], Baikoussis and Verstraelen (1992) investigated the Gauss map of translation surfaces with in 3-space. Particularly, in [19], H. Scherk introduced the special translation surface named Scherk surface which is the only non flat minimal. Then, these type of surfaces have been studied in Euclidean spaces by many geometers with different perspectives (see, [1, 2, 7, 20]). Also, in [3], the authors characterized the translation surfaces in the 3 -dimensional Lorentz-Minkowski space.

In the present study, we consider a spacelike translation surface in Minkowski 4 -space. We define the surface which locally can be written as a monge patch

$$
\phi(u, v)=\left(u, v, f_{1}(u)+g_{1}(v), f_{2}(u)+g_{2}(v)\right),
$$

for some differentiable functions, $f_{i}(u), g_{i}(v), i=1,2$. We characterize such surfaces in terms of their Gaussian curvature and mean curvature functions and give the conditions for such surfaces to become flat and minimal.

\section{Basic Concepts}

The Minkowski 4 -space denoted by $\mathbb{E}_{1}^{4}$ is the space given by the Lorentzian inner product

$$
\langle x, y\rangle=-x_{0} y_{0}+x_{1} y_{1}+x_{2} y_{2}+x_{3} y_{3} \text {. }
$$

Let $S: \phi=\phi(u, v):(u, v) \in D\left(D \subset \mathbb{E}^{2}\right)$ be a spacelike surface in $\mathbb{E}_{1}^{4}$, then $\langle$, induces a Riemannian metric on $S$. Thus, at each point $p$ of a spacelike surface $S$, the following decomposition is available:

$$
\mathbb{E}_{1}^{4}=T_{p}^{\perp} S \oplus T_{p} S,
$$

where the restriction of the metric $\langle$,$\rangle onto the normal space T_{p}^{\perp} S$ and $T_{p} S$ have the signatures $(1,1)$ and $(2,0)$, respectively.

$\tilde{\nabla}$ and $\nabla$ indicate the Levi-Civita connections on $\mathbb{E}_{1}^{4}$ and $S$. Suppose $X$ and $Y$ be vector fields tangent to $M$ and $\xi$ be a normal vector field. The formulas of Weingarten and Gauss decompose the vector fields $\tilde{\nabla}_{X} \xi$ and $\tilde{\nabla}_{X} Y$ into normal and tangent components:

$$
\begin{aligned}
\tilde{\nabla}_{X} \xi & =-A_{\xi} X+D_{X} \xi \\
\tilde{\nabla}_{X} Y & =\nabla_{X} Y+h(X, Y),
\end{aligned}
$$

where $h, D$, and $A_{\xi}$ are the second fundamental form, the normal connection and the shape operator, respectively [6].

The mean curvature vector field $H$ of $S$ can be calculated by $H=\frac{1}{2} t r h$, i.e. given a local orthonormal frame $\{X, Y\}$ of the tangent bundle, $H=\frac{1}{2}((h(X, X)+h(Y, Y))$. 
Let $S: \phi=\phi(u, v):(u, v) \in D\left(D \subset \mathbb{E}^{2}\right)$ be a local parametrization on a spacelike surface in $\mathbb{E}_{1}^{4}$. The tangent space at an arbitrary point $p=\phi(u, v)$ of $S$ is $T_{p} S=\operatorname{span}\left\{\phi_{u}, \phi_{v}\right\}$, where $\left\langle\phi_{u}, \phi_{u}\right\rangle>0,\left\langle\phi_{v}, \phi_{v}\right\rangle>0$. The standard indications $E=\left\langle\phi_{u}, \phi_{u}\right\rangle, F=\left\langle\phi_{u}, \phi_{v}\right\rangle, G=\left\langle\phi_{v}, \phi_{v}\right\rangle$ are used for the coefficients of the first fundamental form

$$
I(\lambda, \mu)=E \lambda^{2}+2 F \lambda \mu+G \mu^{2}, \lambda, \mu \in I R .
$$

[12] Since $I(\lambda, \mu)$ is positive definite, we set $W=\sqrt{E G-F^{2}}$. We choose a normal frame field $\left\{\xi_{1}, \xi_{2}\right\}$ such that $\left\langle\xi_{1}, \xi_{1}\right\rangle=-1,\left\langle\xi_{2}, \xi_{2}\right\rangle=1$, and the quadruple $\left\{\phi_{u}, \phi_{v}, \xi_{1}, \xi_{2}\right\}$ is positively oriented in $\mathbb{E}_{1}^{4}$. Then we have the following derivative formulas:

$$
\begin{aligned}
& \tilde{\nabla}_{\phi_{u}} \phi_{u}=\phi_{u u}=\Gamma_{11}^{1} \phi_{u}+\Gamma_{11}^{2} \phi_{v}-c_{11}^{1} \xi_{1}+c_{11}^{2} \xi_{2}, \\
& \tilde{\nabla}_{\phi_{u}} \phi_{v}=\phi_{u v}=\Gamma_{12}^{1} \phi_{u}+\Gamma_{12}^{2} \phi_{v}-c_{12}^{1} \xi_{1}+c_{12}^{2} \xi_{2}, \\
& \tilde{\nabla}_{\phi_{v}} \phi_{v}=\phi_{v v}=\Gamma_{22}^{1} \phi_{u}+\Gamma_{22}^{2} \phi_{v}-c_{22}^{1} \xi_{1}+c_{22}^{2} \xi_{2},
\end{aligned}
$$

where $\Gamma_{i j}^{k}$ and $c_{i j}^{k},(i, j, k=1,2)$ denote Cristoffel symbols and coefficients of second fundamental form, respectively. Then, these coefficients are given by

$$
\begin{array}{lll}
c_{11}^{1}=\left\langle\phi_{u u}, \xi_{1}\right\rangle, & c_{12}^{1}=\left\langle\phi_{u v}, \xi_{1}\right\rangle, & c_{22}^{1}=\left\langle\phi_{v v}, \xi_{1}\right\rangle, \\
c_{11}^{2}=\left\langle\phi_{u u}, \xi_{2}\right\rangle, & c_{12}^{2}=\left\langle\phi_{u v}, \xi_{2}\right\rangle, & c_{22}^{1}=\left\langle\phi_{v v}, \xi_{2}\right\rangle .
\end{array}
$$

[13] $h$ represents the second fundamental tensor of the surface $S$, then

$$
\begin{aligned}
h\left(\phi_{u}, \phi_{u}\right) & =-c_{11}^{1} \xi_{1}+c_{11}^{2} \xi_{2}, \\
h\left(\phi_{u}, \phi_{v}\right) & =-c_{12}^{1} \xi_{1}+c_{12}^{2} \xi_{2}, \\
h\left(\phi_{v}, \phi_{v}\right) & =-c_{22}^{1} \xi_{1}+c_{22}^{2} \xi_{2} .
\end{aligned}
$$

The second fundamental tensor can be written as

$$
h(X, Y)=-\left\langle A_{\xi_{1}}(X), Y\right\rangle \xi_{1}+\left\langle A_{\xi_{2}}(X), Y\right\rangle \xi_{2} .
$$

[15] The component of $H$ along a given normal connection $N_{k}$, denoted by $H_{k}$, is called the expansion along $\xi_{k}$, i.e., $H_{k}=\left\langle H, \xi_{k}\right\rangle=\frac{\operatorname{tr}\left(A_{\xi_{k}}\right)}{2}$ and we obtain

$$
H_{k}=\frac{c_{11}^{k} G-2 c_{12}^{k} F+c_{22}^{k} E}{2\left(E G-F^{2}\right)}
$$

With regard to the normal basis the mean curvature vector field $H$ becomes

$$
H=-H_{1} \xi_{1}+H_{2} \xi_{2} .
$$

The norm of the mean curvature vector $\|\vec{H}\|$ is called the mean curvature of $S$. If mean curvature vector of a surface is zero, then it is called minimal. 
Gaussian curvature of a regular patch $\phi(u, v)$ can be expressed in terms of the coefficients of the first and second fundamental forms as

$$
K=\frac{-\operatorname{det}\left(A_{\xi_{1}}\right)+\operatorname{det}\left(A_{\xi_{2}}\right)}{W^{2}}=\frac{-c_{11}^{1} c_{22}^{1}+c_{11}^{2} c_{22}^{2}+\left(c_{12}^{1}\right)^{2}-\left(c_{12}^{2}\right)^{2}}{E G-F^{2}} .
$$

A surface $S$ is said to be flat if its Gauss curvature vanishes. [5].

\section{Spacelike Translation Surface in $\mathbb{E}_{1}^{3}$}

The translation surface $S$ determined by curves $\alpha, \beta:(a, b) \rightarrow \mathbb{E}_{1}^{3}$ is the patch

$$
S: \phi(u, v)=\alpha(u)+\beta(v) .
$$

It is the surface formed by moving $\alpha$ parallel to itself in such a way that a point of the curve moves along $\beta[8]$.

A surface that can be generated from two space curves by translating either one of them parallel to itself in such a way that each of its points describes a curve that is a translation of the other curve. For the spacelike surface $S$, both of the generator curves $\alpha(u), \beta(v)$ are spacelike. These curves are defined by the parameterizations

$$
\begin{aligned}
& \alpha(u)=(u, 0, f(u)), \\
& \beta(v)=(0, v, g(v)),
\end{aligned}
$$

where $f(u)$ and $g(v)$ are smooth functions. Thus, the representation of the surface is

$$
\phi(u, v)=(u, v, f(u)+g(v)) .
$$

The natural frame $\left\{\phi_{u}, \phi_{v}\right\}$ is given by

$$
\begin{aligned}
& \phi_{u}=\left(1,0, f^{\prime}(u)\right), \\
& \phi_{v}=\left(0,1, g^{\prime}(v)\right) .
\end{aligned}
$$

Then it follows that the unit normal vector $\xi$ is given by

$$
\xi=\frac{1}{\sqrt{1-f^{\prime 2}+g^{\prime 2}}}\left(f^{\prime},-g^{\prime}, 1\right) \text {. }
$$

The curvatures of the surface in Minkowski 3-space are given by

$$
K=-\frac{f^{\prime \prime} g^{\prime \prime}}{\left(f^{\prime 2}+g^{\prime 2}-1\right)^{2}}
$$

and

$$
H=\frac{\left(1-f^{\prime 2}\right) g^{\prime \prime}+\left(1-g^{\prime 2}\right) f^{\prime \prime}}{2\left(f^{\prime 2}+g^{\prime 2}-1\right)^{\frac{3}{2}}} .
$$


Theorem 3.1. [10] A translation surface parameterized by (3.2) in Minkowski 3-space has constant Gaussian curvature if and only if it is (a part) of a plane or a generalized cylinder and thus, is a flat surface.

Theorem 3.2. [10] A spacelike translation surface in Minkowski 3-space parameterized by (3.2) has mean curvature zero if and only if it is (a part of) either a spacelike plane or the surface of Scherk of the first kind which is parameterized by

$$
\phi(u, v)=\left(u, v, \frac{1}{a} \ln \left|\frac{\cosh (a v)}{\sinh (a u)}\right|\right) \quad \text { with } \tanh ^{2}(a u)+\tanh ^{2}(a v)<1 \text { and } a \in \mathbb{R}_{0} .
$$

\section{Spacelike Translation Surface in $\mathbb{E}_{1}^{4}$}

Definition 4.1. A surface can be determined by the curves $\alpha, \beta:(a, b) \rightarrow \mathbb{E}_{1}^{4}$ is the patch

$$
\begin{array}{rll}
\phi & : & \mathbb{E}^{2} \rightarrow \mathbb{E}_{1}^{4} \\
\phi(u, v) & =\alpha(u)+\beta(v) .
\end{array}
$$

If the generating curves $\alpha(u)$ and $\beta(v)$ are space curves has the parameterizations

$$
\begin{aligned}
\alpha(u) & =\left(u, 0, f_{1}(u), f_{2}(u)\right), \\
\beta(v) & =\left(0, v, g_{1}(v), g_{2}(v)\right),
\end{aligned}
$$

then this surface is still called translation surface in $\mathbb{E}_{1}^{4}$. Thus, the translation surface is defined by the patch

$$
\phi(u, v)=\left(u, v, f_{1}(u)+g_{1}(v), f_{2}(u)+g_{2}(v)\right) .
$$

Let the surface $S$ be spacelike, then both of the generator curves $\alpha(u), \beta(v)$ are spacelike. The first partial derivatives of $\phi(u, v)$ are given by

$$
\begin{aligned}
& \phi_{u}=\left(1,0, f_{1}^{\prime}(u), f_{2}^{\prime}(u)\right), \\
& \phi_{v}=\left(0,1, g_{1}^{\prime}(v), g_{2}^{\prime}(v)\right) .
\end{aligned}
$$

Hence, the coefficients of the first fundamental form of the surface as we can find

$$
\begin{aligned}
& E=\left\langle\phi_{u}, \phi_{u}\right\rangle=-1+\left(f_{1}^{\prime}\right)^{2}+\left(f_{2}^{\prime}\right)^{2}, \\
& F=\left\langle\phi_{u}, \phi_{v}\right\rangle=f_{1}^{\prime} g_{1}^{\prime}+f_{2}^{\prime} g_{2}^{\prime}, \\
& G=\left\langle\phi_{v}, \phi_{v}\right\rangle=1+\left(g_{1}^{\prime}\right)^{2}+\left(g_{2}^{\prime}\right)^{2},
\end{aligned}
$$


where $\langle$,$\rangle is Lorentzian inner product in \mathbb{E}_{1}^{4}$. Since the first fundamental form is positive definite, we set $W=\sqrt{E G-F^{2}}$.

The second partial derivatives of $\phi(u, v)$ are expressed as

$$
\begin{aligned}
\phi_{u u} & =\left(0,0, f_{1}^{\prime \prime}(u), f_{2}^{\prime \prime}(u)\right), \\
\phi_{u v} & =(0,0,0,0), \\
\phi_{v v} & =\left(0,0, g_{1}^{\prime \prime}(v), g_{2}^{\prime \prime}(v)\right) .
\end{aligned}
$$

It follows that chosen normal frame field $\left\{\xi_{1}, \xi_{2}\right\}$

$$
\begin{aligned}
& \xi_{1}=\frac{1}{\sqrt{|A|}}\left(f_{1}^{\prime}(u),-g_{1}^{\prime}(v), 1,0\right), \\
& \xi_{2}=\frac{1}{\sqrt{A D}}\left(A f_{1}^{\prime}(u)-B f_{2}^{\prime}(u), B g_{1}^{\prime}(v)-A g_{2}^{\prime}(v),-B, A\right),
\end{aligned}
$$

where

$$
\begin{aligned}
& A=1-\left(f_{1}^{\prime}\right)^{2}+\left(g_{1}^{\prime}\right)^{2}, \\
& B=-f_{1}^{\prime} f_{2}^{\prime}+g_{1}^{\prime} g_{2}^{\prime}, \\
& C=1-\left(f_{3}^{\prime}\right)^{2}+\left(g_{3}^{\prime}\right)^{2}, \\
& D=A C-B^{2},
\end{aligned}
$$

and by the use of (4.4) and (4.5), the functions $c_{i j}^{k},(i, j, k=1,2)$ are given by

$$
\begin{aligned}
& c_{11}^{1}=\frac{f_{1}^{\prime \prime}}{\sqrt{|A|}}, \quad c_{22}^{1}=\frac{g_{1}^{\prime \prime}}{\sqrt{|A|}}, \\
& c_{12}^{1}=c_{12}^{2}=0, \\
& c_{11}^{2}=\frac{A f_{2}^{\prime \prime}-B f_{1}^{\prime \prime}}{\sqrt{A D}}, \\
& c_{22}^{2}=\frac{A g_{2}^{\prime \prime}-B g_{1}^{\prime \prime}}{\sqrt{A D}} .
\end{aligned}
$$

Using Gram-Schmidt orthonormalization method for the spacelike vector fields $\phi_{u}$ and $\phi_{v}$, we get orthonormal tangent vectors

$$
\begin{aligned}
X & =\frac{\phi_{u}}{\sqrt{E}}, \\
Y & =\frac{\sqrt{E}}{W}\left(\phi_{v}-\frac{F}{E} \phi_{u}\right) .
\end{aligned}
$$


By the use of $(2.3),(2.4),(2.5)$ and (4.7), the shape operator matrices can be written as

$$
\begin{aligned}
& A_{\xi_{1}}=\frac{1}{E \sqrt{|A|}}\left[\begin{array}{cc}
f_{1}^{\prime \prime} & \frac{-f_{1}^{\prime \prime} F}{W} \\
\frac{-f_{1}^{\prime \prime} F}{W} & \frac{g_{1}^{\prime \prime} E^{2}+f_{1}^{\prime \prime} F^{2}}{W^{2}}
\end{array}\right], \\
& A_{\xi_{2}}=\frac{1}{E \sqrt{|A D|}}\left[\begin{array}{cc}
A f_{2}^{\prime \prime}-B f_{1}^{\prime \prime} & \frac{-\left(A f_{2}^{\prime \prime}-B f_{1}^{\prime \prime}\right) F}{W} \\
\frac{-\left(A f_{2}^{\prime \prime}-B f_{1}^{\prime \prime}\right) F}{W} & \frac{\left(A g_{2}^{\prime \prime}-B g_{1}^{\prime \prime}\right) E^{2}+\left(A f_{2}^{\prime \prime}-B f_{1}^{\prime \prime}\right) F^{2}}{W^{2}}
\end{array}\right] .
\end{aligned}
$$

Theorem 4.1. Let $S$ be a spacelike translation surface in $\mathbb{E}_{1}^{4}$. Then Gaussian curvature of $S$ is given by

$$
K=\frac{f_{1}^{\prime \prime} g_{1}^{\prime \prime} C-\left(f_{1}^{\prime \prime} g_{2}^{\prime \prime}+g_{1}^{\prime \prime} f_{2}^{\prime \prime}\right) B+f_{2}^{\prime \prime} g_{2}^{\prime \prime} A}{W^{2} D} .
$$

Proof. By the use of the equations (2.8) and (4.6), we get the result.

Theorem 4.2. Let $S$ be a spacelike translation surface parameterized by (4.1). Then $S$ is a flat surface if and only if it is (a part) of a plane or a generalized cylinder given by

$$
\phi(u, v)=\left(u, 0, f_{1}(u)+a_{1}, f_{2}(u)+a_{2}\right)+v\left(0,1, b_{1}, b_{2}\right)
$$

or

$$
\phi(u, v)=\left(0, v, g_{1}(v)+c_{1}, g_{2}(v)+c_{2}\right)+u\left(1,0, d_{1}, d_{2}\right),
$$

where $a_{i}, b_{i}, c_{i}, d_{i}(i=1,2)$ are real constants.

Proof. Let $S$ be a spacelike translation surface parameterized by (4.1). If the Gaussian curvature of the surface is zero, then we get $f_{i}^{\prime}=0, g_{i}^{\prime}=0$, or $f_{i}^{\prime \prime}=0$, or $g_{i}^{\prime \prime}=0$ $(i=1,2)$. For the first case we obtain a plane. For the second and third case, we get generalized cylinders with the parameterizations (4.10) and (4.11), respectively. This completes the proof.

Theorem 4.3. Let $S$ be a spacelike translation surface with the parametrization (4.1) in $\mathbb{E}_{1}^{4}$. Then the mean curvature vector field is given by

$$
\vec{H}=-\frac{f_{1}^{\prime \prime} G+g_{1}^{\prime \prime} E}{2 \sqrt{|A|} W^{2}} \xi_{1}+\frac{G\left(f_{2}^{\prime \prime} A-f_{1}^{\prime \prime} B\right)+E\left(g_{2}^{\prime \prime} A-g_{1}^{\prime \prime} B\right)}{2 \sqrt{A D} W^{2}} \xi_{2} .
$$

Proof. By the use of the equations (2.6), (2.7) and (4.6), we obtain the desired result. 
Proposition 4.1. Let $S$ be a spacelike translation surface with the parametrization (4.1) in $\mathbb{E}_{1}^{4}$. Then $S$ is a minimal surface if and only if

$$
-\frac{f_{i}^{\prime \prime}}{E}=\frac{g_{i}^{\prime \prime}}{G}=c_{i}, \quad i=1,2
$$

where $c_{i},(i=1,2)$ are real constants.

Proof. Let $S$ be a spacelike translation surface with the parametrization (4.1). If $S$ is minimal, then the mean curvature vector field is zero, namely the components of $\vec{H}$ are zero. From the equation (4.12), we get the result.

Theorem 4.4. Let $S$ be a spacelike translation surface in $\mathbb{E}_{1}^{4}$ with the parametriza-

tion (4.1). Then $S$ is a minimal surface if and only if either $S$ is a plane or the functions $f_{i}(u), g_{i}(v)$ are defined by

$$
f_{i}(u)=\frac{c_{i}}{c_{1}^{2}+c_{2}^{2}}(\ln (\cos \sqrt{d} u)-b u)+k_{i} u, d>0 \quad i=1,2
$$

or

$$
f_{i}(u)=-\frac{c_{i}}{c_{1}^{2}+c_{2}^{2}}(\ln (\cosh \sqrt{|d|} u)+b u)+k_{i} u, d<0 \quad i=1,2
$$

and

$$
g_{i}(v)=-\frac{c_{i}}{c_{1}^{2}+c_{2}^{2}}\left(\ln \left(\cos \sqrt{d^{\prime}} v\right)+b^{\prime} v\right)+l_{2} v, \quad i=1,2
$$

where $d^{\prime}$ positive and $b, b^{\prime}, c_{i}, d, k_{i}, l_{i}$ are real constants.

Proof. Let $S$ be a translation surface in $\mathbb{E}_{1}^{4}$ which satisfies the equation (4.13). Then

$$
-\frac{f_{i}^{\prime \prime}(u)}{-1+\left(f_{1}^{\prime}(u)\right)^{2}+\left(f_{2}^{\prime}(u)\right)^{2}}=\frac{g_{i}^{\prime \prime}(v)}{1+\left(g_{1}^{\prime}(u)\right)^{2}+\left(g_{2}^{\prime}(u)\right)^{2}}=c_{i} .
$$

We know that the variables $u$ and $v$ are independent. Hence, left and right side of the equation must be constant. Thus, we have

$$
\begin{aligned}
& f_{i}^{\prime \prime}(u)=-c_{i}\left(-1+\left(f_{1}^{\prime}(u)\right)^{2}+\left(f_{2}^{\prime}(u)\right)^{2}\right), \\
& g_{i}^{\prime \prime}(v)=c_{i}\left(1+\left(g_{1}^{\prime}(u)\right)^{2}+\left(g_{2}^{\prime}(u)\right)^{2}\right) .
\end{aligned}
$$

Suppose $c_{i}=0, i=1,2$, then we obtain $f_{i}(u)=a_{i} u+b_{i}$ and $g_{i}(v)=c_{i} v+d_{i}$. As a result of this, $M$ is a plane in Minkowski 4 -space. Furthermore, we assume that $c_{1} \neq 0$, by dividing the equations (4.14) by the same equations for $i=1$, we get

$$
\frac{f_{i}^{\prime \prime}(u)}{f_{1}^{\prime \prime}(u)}=\frac{g_{i}^{\prime \prime}(v)}{g_{1}^{\prime \prime}(v)}=\frac{c_{i}}{c_{1}}, \quad i=1,2
$$


Therefore

$$
\begin{aligned}
f_{i}^{\prime \prime}(u) & =\frac{c_{i}}{c_{1}} f_{1}^{\prime \prime}(u), \\
g_{i}^{\prime \prime}(v) & =\frac{c_{i}}{c_{1}} g_{1}^{\prime \prime}(v),
\end{aligned}
$$

and then

$$
\begin{aligned}
f_{i}^{\prime}(u) & =\frac{c_{i}}{c_{1}} f_{1}^{\prime}(u)+k_{i} \\
g_{i}^{\prime}(v) & =\frac{c_{i}}{c_{1}} g_{1}^{\prime}(v)+l_{i}
\end{aligned}
$$

where $k_{i}$ and $l_{i}$ are real constants for $i=1,2$ with $k_{1}=l_{1}=0$. Consider the equations (3.2) for $i=1$ :

$$
\begin{aligned}
& f_{1}^{\prime \prime}(u)=-c_{1}\left(-1+\left(f_{1}^{\prime}(u)\right)^{2}+\left(f_{2}^{\prime}(u)\right)^{2}\right), \\
& g_{1}^{\prime \prime}(v)=c_{1}\left(1+\left(g_{1}^{\prime}(u)\right)^{2}+\left(g_{2}^{\prime}(u)\right)^{2}\right)
\end{aligned}
$$

and substitute (4.15) into these equations. We have

$$
\begin{aligned}
& f_{1}^{\prime \prime}(u)=-\frac{c_{1}^{2}+c_{2}^{2}}{c_{1}}\left(f_{1}^{\prime}\right)^{2}-2 c_{2} k_{2} f_{1}^{\prime}-c_{1}\left(k_{2}^{2}-1\right), \\
& g_{1}^{\prime \prime}(v)=\frac{c_{1}^{2}+c_{2}^{2}}{c_{1}}\left(g_{1}^{\prime}\right)^{2}+2 c_{2} l_{2} g_{1}^{\prime}+c_{1}\left(l_{2}^{2}+1\right) .
\end{aligned}
$$

Then taking

$$
\begin{aligned}
f_{1}^{\prime}(u) & =p, \quad g_{1}^{\prime}(v)=q, \quad a=\frac{c_{1}^{2}+c_{2}^{2}}{c_{1}}, \quad b=c_{2} k_{2}, \\
c & =c_{1}\left(k_{2}^{2}-1\right), \quad b^{\prime}=c_{2} l_{2}, \quad c^{\prime}=c_{1}\left(l_{2}^{2}+1\right),
\end{aligned}
$$

we obtain the differential equations

$$
\begin{aligned}
& \frac{d p}{d u}=-\left(a p^{2}+2 b p+c\right) \\
& \frac{d q}{d v}=\left(a q^{2}+2 b^{\prime} q+c^{\prime}\right)
\end{aligned}
$$

or we can write

$$
\begin{aligned}
& \frac{d p}{d u}=-\frac{1}{a}\left[(a p+b)^{2}+a c-b^{2}\right], \\
& \frac{d q}{d v}=\frac{1}{a}\left[\left(a q+b^{\prime}\right)^{2}+a c^{\prime}-b^{\prime 2}\right] .
\end{aligned}
$$

Put $d=a c-b^{2}$ and $d^{\prime}=a c^{\prime}-b^{\prime 2}$, then

$$
\begin{aligned}
d & =c_{1}^{2}\left(k_{2}^{2}-1\right)-c_{2}^{2}, \\
d^{\prime} & =c_{2}^{2}\left(l_{2}^{2}+1\right)+c_{2}^{2},
\end{aligned}
$$


where $d$ is constant and $d^{\prime}$ is positive constant. Assume both of them are positive, we get

$$
\begin{aligned}
& p=f_{1}^{\prime}(u)=\frac{-b-\sqrt{d} \tan (\sqrt{d} u)}{a}, \\
& q=g_{1}^{\prime}(v)=\frac{-b^{\prime}+\sqrt{d^{\prime}} \tan \left(\sqrt{d^{\prime}} v\right)}{a} .
\end{aligned}
$$

If $d$ is negative, then

$$
p=f_{1}^{\prime}(u)=\frac{-b+\sqrt{|d|} \tanh (\sqrt{|d|} u)}{a} .
$$

Using these result and equality (4.15), the other functions are

$$
\begin{aligned}
& f_{2}^{\prime}(u)=-\frac{c_{2}}{c_{1}^{2}+c_{2}^{2}}(\sqrt{d} \tan (\sqrt{d} u)+b)+k_{2} u, d>0, \\
& g_{2}^{\prime}(v)=\frac{c_{2}}{c_{1}^{2}+c_{2}^{2}}\left(\sqrt{d^{\prime}} \tan \left(\sqrt{d^{\prime}} v\right)-b^{\prime}\right)+l_{2} v, d^{\prime}>0,
\end{aligned}
$$

or

$$
f_{2}^{\prime}(u)=\frac{c_{2}}{c_{1}^{2}+c_{2}^{2}}(\sqrt{|d|} \tanh (\sqrt{|d|} u)-b)+k_{2} u, d<0 .
$$

Consequently, we have all the solutions

$$
f_{i}(u)=\frac{c_{i}}{c_{1}^{2}+c_{2}^{2}}(\ln (\cos \sqrt{d} u)-b u)+k_{i} u, d>0
$$

or

$$
f_{i}(u)=-\frac{c_{i}}{c_{1}^{2}+c_{2}^{2}}(\ln (\cosh \sqrt{|d|} u)+b u)+k_{i} u, d<0
$$

and

$$
g_{i}(v)=-\frac{c_{i}}{c_{1}^{2}+c_{2}^{2}}\left(\ln \left(\cos \sqrt{d^{\prime}} v\right)+b^{\prime} v\right)+l_{2} v, d^{\prime}>0
$$

for $i=1,2$

Example 4.1. The surface given by the parametrization

$$
\phi(u, v)=(u, v,-3 u+2 v-\ln (\cosh 2 u \cos 3 v),-2 u+v-2 \ln (\cosh 2 u \cos 3 v)
$$

is minimal in Minkowski 4-space. 


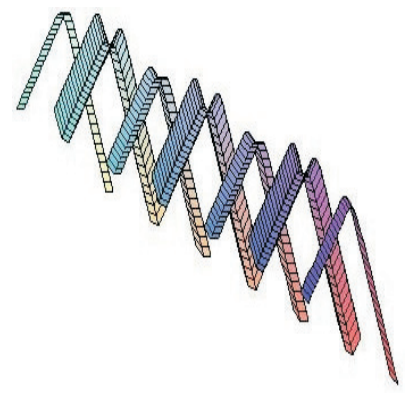

FIG. 4.1: 3D model obtained by the projection of Spacelike Minimal Surface (4.16)

\section{REFEREN CES}

1. K. Arslan, B. Bayram, B. Bulca And G. ÖZtürk: On translation surfaces in 4-dimensional Euclidean space. Acta Et Commentationes Universitatis Tartuensis De Mathematics. 20(2016),123-133.

2. B. Bulca And K. Arslan: Surfaces Given with the Monge Patch in $\mathbb{E}^{4}$.Journal of Mathematical Physics, Analysis, Geometry,9(2013), 435-447.

3. Сh. BABA-HAmed, M. BeKkAR AND H. Zoubir: Translation surfaces in the threedimensional Lorentz-Minkowski space satisfying $\Delta r_{i}=\lambda_{i} r_{i}$. Int. Journal of Math. Analysis, 4 (2010), 797-808.

4. C. Baikoussis and L. Verstraelen: On Gauss map of translation surfaces. Rend. Sem. Mat. Messina Ser II, 2015.

5. B.Y. Chen: Geometry of Submanifolds.Dekker, New York 1973.

6. B.Y. Chen, J. VAN DER VEKEN: Marginally trapped surfaces in Lorentzian space forms with positive relative nullity. Class. Quantum Grav. 24 (2007), 551-563.

7. M. ÇETin, Y. Tuncer AND N. Ekmekci: Translation surfaces in Euclidean 3-space, Int. Journal of Physical and Mathematical Science 2 (2011), 49-56.

8. F. Dillen, L. Verstraelen, L. Vrancken, and G. Zafindratafa: Classification of Polynomial Translation Hypersurfaces of Finite Type, Results in Math. 27 (1995), 244-249.

9. G. Forni And C. Matheus: Introduction to Teichmüller theory and its applications to dynamics of interval exchange transformations, flows on surfaces and billiards J. Mod. Dyn., 8 (2014), 271-416.

10. W. Geomans: Surfaces in three-dimensional Euclidean and Minkowski space, in particular a study of Weingarten surfaces. PhD Thesis, Katholieke Universiteit LeuvenFaculty of Science, 2010.

11. J. Glymph, D. Schelden, C. Ceccato, J. Mussel, H. Schober: A parametric strategy for free-form glass structures using quadrilateral planar facets. Automation in Construction, 13 (2004), 187-202.

12. G. Gancgev and V. Milousheva: An invariant theory of Marginally Trapped Surfaces in four-dimensional Minkowski space. Journal of Mathematical Physics,53 (2012), 033705 . 
13. G. Gancgev And V. Milousheva: An invariant theory of spacelike surfaces in the four-dimensional Minkowski space. Pliska Stud. Math. Bulgar. 21 (2012), 177-200.

14. J.M. Gutierrez Nunez, M.C. Romero Fuster and F. Sanchez-Bringas: Codazzi Fields on Surfaces Immersed in Euclidean 4-spaces. OsAKA J. MATH. 45 (2008), 877-894.

15. S. HeAsen AND M. ORTEGa: Marginally trapped surfaces in Minkowski 4-space invariant under a rotation subgroup of the Lorentz group. Gen Relativ Gravit. 41 (2009), 1819-1834.

16. İ. KIŞI AND G. ÖzTüRK: A new approach to canal surface with parallel transport frame. Int. J. Geom. Methods Mod. Phys. 14 (2017), 1750026.

17. R. Osserman: Global properties of minimal surfaces in $\mathbb{E}^{3}$ and $\mathbb{E}^{n}$. Ann. OF Math. 80 (1964), 340-364.

18. J. Perez: A new golden age of minimal surfaces. Notices of the AMS 64 (2017), $347-358$

19. H.F. ScheRK: Bemerkungen über die kleinste Flache innerhalb gegebener Grenzen. J. Reine Angew. Math. (1835), 185-208.

20. L. Verstraelen, J. Walrave and S. Yaprak: The Minimal Translation Surface in Euclidean Space. Soochow J. MATH. 20 (1994), 77-82.

Sezgin Büyükkütük

Gölcük Vocational School of Higher Education

Kocaeli University

41380 Kocaeli, Turkey

sezgin. buyukkutuk@kocaeli.edu.tr

Günay Öztürk

Department of Mathematics

Izmir Demokrasi University

Izmir, Turkey

gunay.ozturk@idu.edu.tr 